\title{
Preliminary Modeling to Support the TREAT Hodoscope System for Fuel Motion Monitoring
}

\section{Physics of Reactors Conference (PHYSOR 2016)}

Scott J. Thompson, James T. Johnson, Robert S. Schley, Clive H. Townsend, David L. Chichester

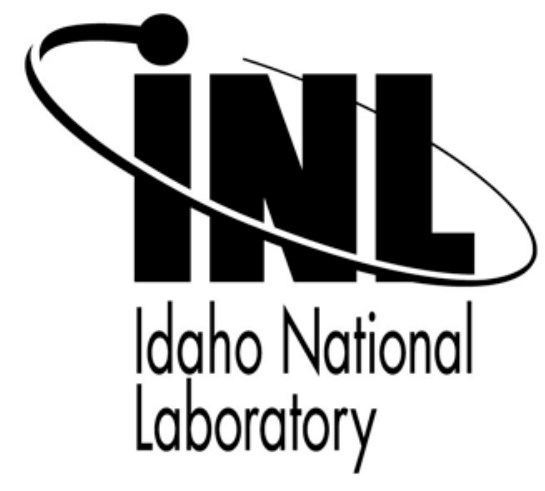

This is a preprint of a paper intended for publication in a journal or proceedings. Since changes may be made before publication, this preprint should not be cited or reproduced without permission of the author. This document was prepared as an account of work sponsored by an agency of the United States Government. Neither the United States Government nor any agency thereof, or any of their employees, makes any warranty, expressed or implied, or assumes any legal liability or responsibility for any third party's use, or the results of such use, of any information, apparatus, product or process disclosed in this report, or represents that its use by such third party would not infringe privately owned rights. The views expressed in this paper are not necessarily those of the United States Government or the sponsoring agency. 



\title{
PRELINARY MODELING TO SUPPORT THE TREAT HODOSCOPE SYSTEM FOR FUEL MOTION MONITORING
}

\author{
Scott J. Thompson, James T. Johnson, Robert S. Schley, Clive H. Townsend, \\ and David L. Chichester \\ Nuclear Nonproliferation Division \\ Idaho National Laboratory, Idaho Falls, Idaho, USA \\ scott.thompson@inl.gov \\ clive.townsend@inl.gov \\ david.chichester@inl.gov
}

\begin{abstract}
Fuel motion is monitored in real time at the Transient Reactor Test Facility (TREAT) using a direct-viewing hodoscope that monitors fast neutrons emitted from test fuel undergoing fission during transients. The hodoscope system is now being refurbished, after over twenty years, to once again support research and development activities examining the survival and failure modes of advanced nuclear reactor fuel systems under accident conditions. An important part of this activity is the development of high-fidelity modeling tools to predict the hodoscope's response to different fuel systems, in different test vehicles, throughout different transient cycles. Initial work has involved the collection of archival data related to how the hodoscope currently at TREAT was manufactured and installed, and the use of this data to create a preliminary radiation transport model using MCNP6. This model is now undergoing refinement to improve its fidelity and to permit its integration with other tools under development to model TREAT transients. The model will be used to study how fast-neutrons measured in the hodoscope's detectors relate to the fission of nuclear material in a test vehicle as a function of location within the vehicle and reactor power. The goal is to develop a time-dependent coupling factor that will relate the measured data to the fuel mass present within each pixel of the hodoscope's field of view. This paper presents the current status of the TREAT hodoscope refurbishment program and modeling activities related to interpreting hodoscope data and inferring fuel motion during transients.
\end{abstract}

Key Words: TREAT, hodoscope, fast neutron, transient modeling

\section{INTRODUCTION}

Work is now underway at Idaho National Laboratory (INL) to restart the Transient Reactor Test Facility (TREAT). TREAT is a graphite-moderated, air-cooled reactor specifically designed for transient testing. It is fueled with a $19 \times 19$ array of $100 \mathrm{~mm} \times 100 \mathrm{~mm}$ square fuel assemblies of highly-enriched uranium dioxide dispersed through a graphite and carbon-matrix. The fueled region of the core is $1210-\mathrm{mm}$ tall; there are 650 -mm tall graphite reflector components above and below the core and $280 \mathrm{~mm}$ of graphite reflectors on its sides.[1] The TREAT reactor's purpose is to test irradiated nuclear reactor fuel under off-normal and accident conditions to: a) provide insight into how and why reactor fuel fails during accidents; b) determine performance margins for nuclear fuel, and c) test and evaluate new fuel systems. 
A key measurement performed during these tests is monitoring the movement and relocation of fuel, during high-power transients, due to internal heating from fission. Understanding fuel measurement and relocation is important because it provides insights into how fuel interacts with cladding, fuel-assembly structural materials, and coolant. This allows analysts to estimate fuel fragmentation modes and thresholds and to understand how different accident scenarios progress. A comprehensive understanding of different accident scenarios is needed to evaluate the importance and vulnerability of different reactor design concepts and systems.

To perform experiments one or two fuel slots at the center of the core are loaded with an experiment capsule that contains the test fuel. During tests the reactor produces one high-power transient pulse, with a peak power of up to $16,000 \mathrm{MW}$, which can be tuned to represent different accident scenarios. Experiment capsules can hold single fuel pins or arrays of pins and have been designed to study fuel in air, water, or sodium, and with or without coolant flow.

The principle measurement tool used at TREAT is the TREAT fast-neutron hodoscope, which is used for fuel motion monitoring throughout the duration of a transient.[1] The hodoscope is a direct-view imaging system that provides time and spatial resolution of fuel motion during transients, and in-place measurement of fuel distribution before and after experiments (Figure 1).[2] The hodoscope consists of a multi-channel slot-collimator subsystem that is inserted into the reactor's biological shield, a fast-neutron detector array arranged at the rear-face of the collimator, and a data acquisition system. During an experiment, thermalized neutrons from the reactor induce fission within the test fuel. Fast neutrons generated in the test fuel escape the core, travel through the hodoscope and are detected. Those neutrons which traveled through a single slot in the hodoscope and are detected can then be correlated to their origin within the experiment itself. The detector system time stamps each detection event allowing for fuel motion monitoring during transients.

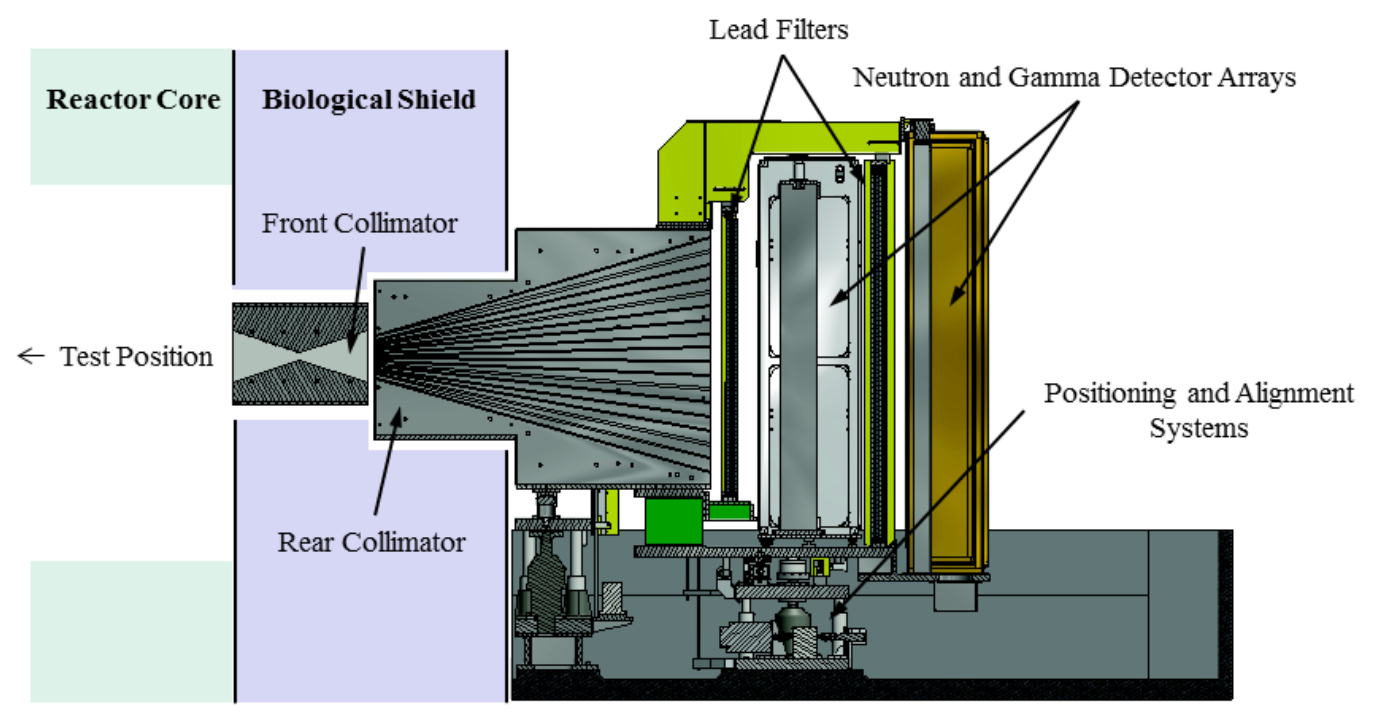

Figure 1. A cross-section drawing of the 1.2-m collimator hodoscope system demonstrating how the components interface with the TREAT reactor. 
As a part of the reactor restart program work is also underway to refurbish the hodoscope, including its detectors. One type of detector used in the hodoscope is a fast-neutron scintillator (called a "Hornyak" button) originally developed in 1952.[3,4] The TREAT hodoscope can hold an array of 360 Hornyak-button detectors. Photographs of a fast-neutron scintillator detector assembly, and an individual button scintillator, are shown in Figure 2.
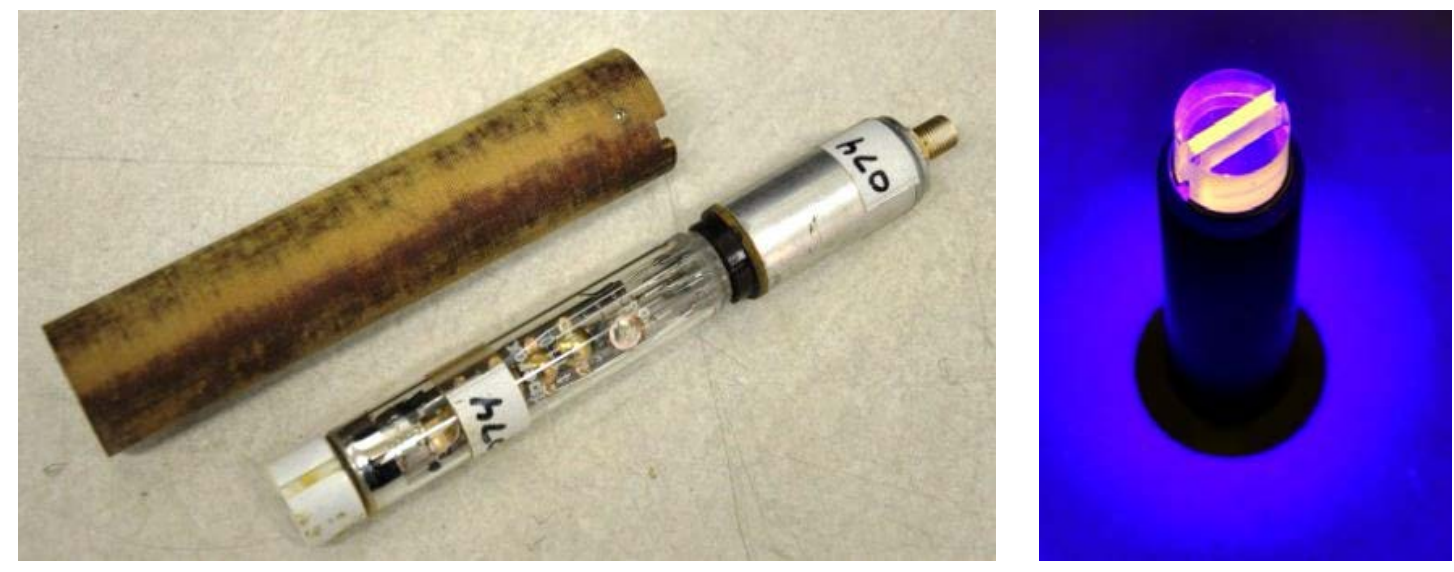

Figure 2. The image on the left shows one of the original Hornyak-button detector assemblies used at TREAT. The image to the right shows a Hornyak-button scintillator, cleaned and polished, under ultraviolet illumination.

This paper is a report on recent research activities underway at INL to model and predict the expected performance and operation capabilities of the TREAT hodoscope for monitoring fuel motion during transient tests. Initial background activities have focused on gathering and organizing engineering drawings, correspondence, and reactor documentation to develop an accurate understanding of the as-built configuration of the hodoscope currently installed at TREAT. As the TREAT facility was placed in standby approximately two decades ago, regaining continuity of knowledge and institutional understanding of some of its instrumentation and research systems have been significant challenges.

Simulation and modeling efforts to-date have been focused in two thrust areas with the primary goal of connecting time- and location-dependent fissioning mass quantities in the experimental test capsule to a final multichannel detection signal from the hodoscope system. The first area of work is aimed at characterizing the neutron and gamma flux from both the experiment and the reactor background at the point of detection. The second thrust area is detector focused with the intent of characterizing the intensity and shape of the light yield from the fast-neutron button detectors.

\section{TREAT HODOSCOPE FLUX MODELING}

The principal components of the TREAT hodoscope are the two immense collimators designed to limit the view of each of the 360 detector positions to single image pixel of viewing area at the test position. The non-occluding front collimator has two opposing focal shields to help confine the 
neutron path through a common point. The rear collimator consists of 20 laminated sheets of steel with 18 angled slots each (360 total pathways) for particle passage to the detectors, which are approximately 4.5 meters from the test item. These slots fan out to accommodate the 360 detector array; cross-talk between channels is minimized due to the long collimator, fast neutrons must traverse a minimum of $1.2 \mathrm{~m}$ of steel to enter an adjacent channel.[5] A three-dimensional drawing of the rear collimator is shown in Figure 3 that provides the external dimensions of this component. Finally, lead panels provide photon shielding to help improve the signal-to-background ratio of the system.

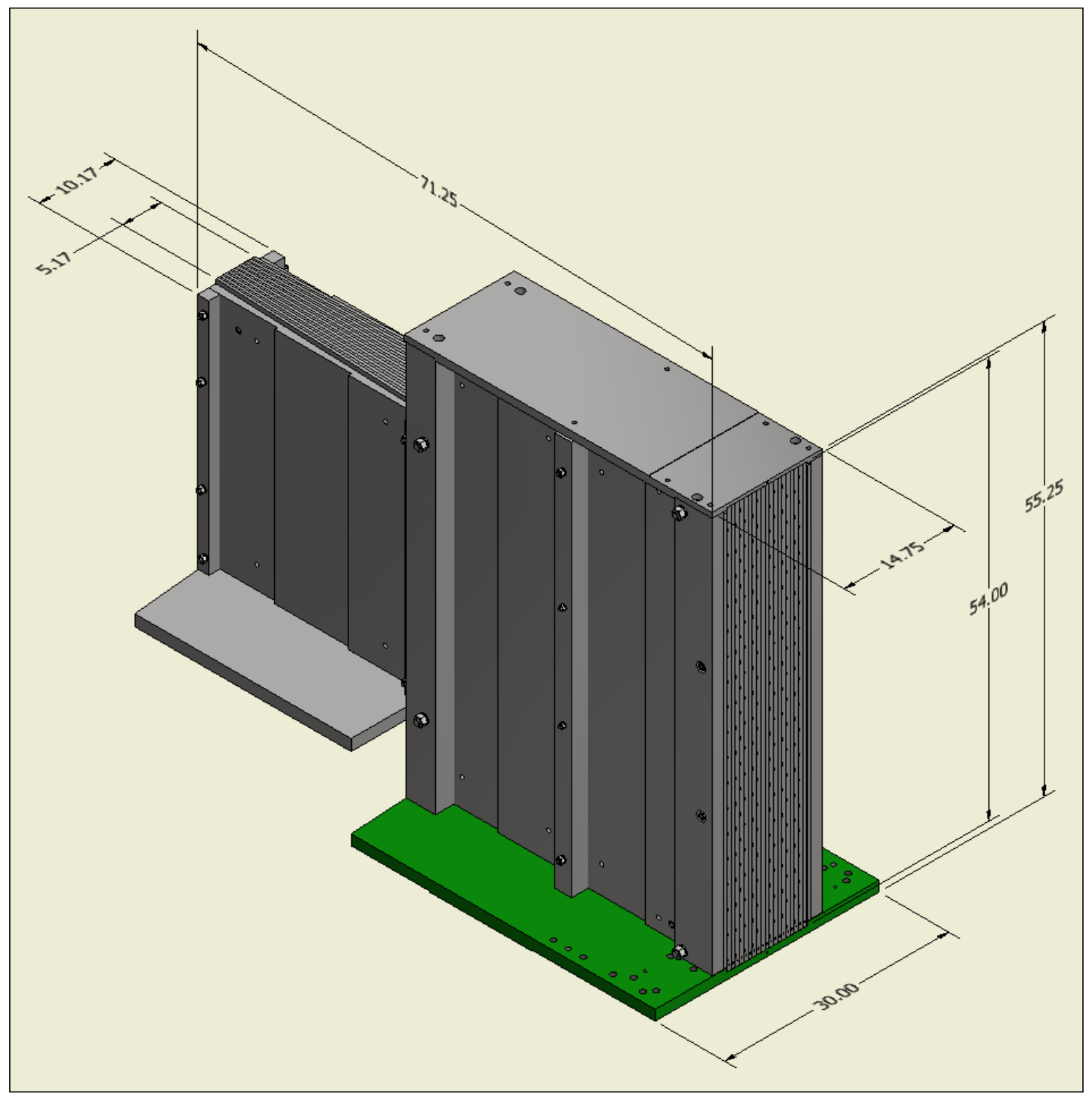

Figure 3. Engineering drawing of the currently installed version of the TREAT hodoscope rear collimator. Dimensions provided are in units of inches. 
A Monte Carlo n-Particle (MCNP6) model of the hodoscope system's front and rear collimators, lead photon filters, and other key components was recently created; a cross-section of this model is shown in Figure 4.[6] The particle-occluding nature of the TREAT collimator necessitates the heavy application of variance reduction techniques in the simulation and modeling efforts for statistical convergence of the Monte-Carlo calculated flux values at the detector position. One of the most basic mechanisms to aid this statistical convergence is to break the problem into successively dependent segments. This also allows for the use of pre-existing, validated models of the TREAT reactor in hodoscope calculations.

Work was done to couple an existing model of the TREAT reactor to the front-end hodoscope model to calculate the resulting detector-position fluxes of neutrons from both induced fissions at the test position as well as the operational background of the reactor. The MCNP6 functionality to track particles crossing a surface and tally their direction, energy, and other relevant parameters was used to facilitate the coupling. Information for each particle crossing the surface where the two models interface is written to a surface-crossing history file using the Surface Source Write (SSW) functionality. This feature is typically used in the execution of a KCODE calculation but may also be used with particles originating from a standard SDEF source definition. It is then possible to apply the information saved in the history file as a position and angular-dependent source definition to sample from on subsequent runs. This is done using the Surface Source Read (SSR) card. Special care must be taken to account for the albedo effects of structural components downstream from the surface of interest. In order to accomplish this, both the reactor and hodoscope models have been extended beyond the region of interface to include structural components of the complimenting model.

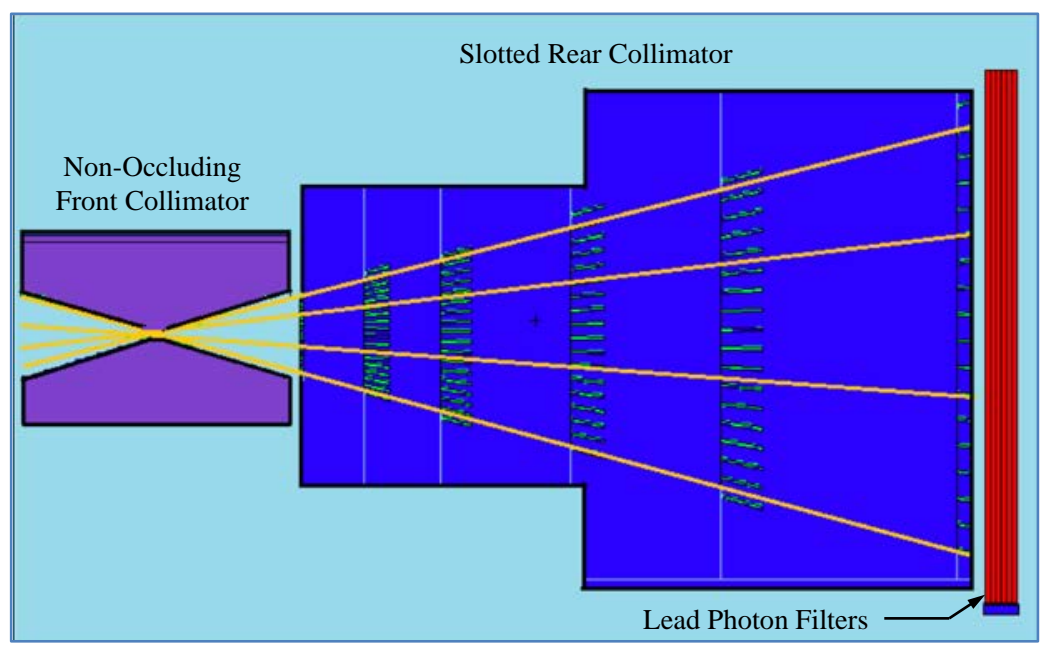

Figure 4. MCNP6-generated cross-sectional drawing of the TREAT hodoscope radiation transport model.

Another goal of this work is to determine an energy, position, and angular-dependent hodoscope collimator response function that can be applied to incident flux values in future calculations, essentially bypassing the computational overhead brought about from the variance reduction needed to transport particles through the collimator components. To this end, further simulations have been 
initiated to characterize the energy-dependent fraction of neutrons that traverse the full extent of the collimators and filters and understand the umbra, penumbra, and antumbra produced by these components. Once a response is determined it can be convolved with a detector array response, described in the following section, to produce an overall hodoscope system response.

\section{HODOSCOPE DETECTOR MODELING}

A complete model of the TREAT hodoscope system must include the physics involved in converting neutron and gamma fluxes into a measureable signal. For the scintillation-based Hornyak buttons this means converting the kinetic energies of recoiling particles and nuclei in the $\mathrm{ZnS}(\mathrm{Ag})$ material into optical wavelength light to determine an energy-dependent response. These calculations are currently being performed using two different tools in tandem to garner a complete understanding.

MCNPX-PoliMi is an extension of the MCNPX v2.7 code commonly used for problems that involve scintillators and coincidence based detection schemes.[7] This code has the ability to produce a summary file of the neutron and photon interactions in modeled detector volumes, much like a heavily-filtered and human-readable version of the standard MCNP ptrac file. The MCNPX-PoliMi package provides a suite of tunable algorithms to convert the summary file into detector pulses, most commonly used to convert energy deposition into units of light for organic scintillators. A MCNPX-PoliMi model of a TREAT Hornyak-button detector has been created to characterize light generation in the detector. A drawing of this model is shown in Figure 5. The post-processing algorithms are currently being enhanced to include $\mathrm{ZnS}(\mathrm{Ag})$ materials and to account for recoiling protons that originate in the pure Lucite waveguide portions of the detector before entering the ZnS(Ag)-Lucite compound.

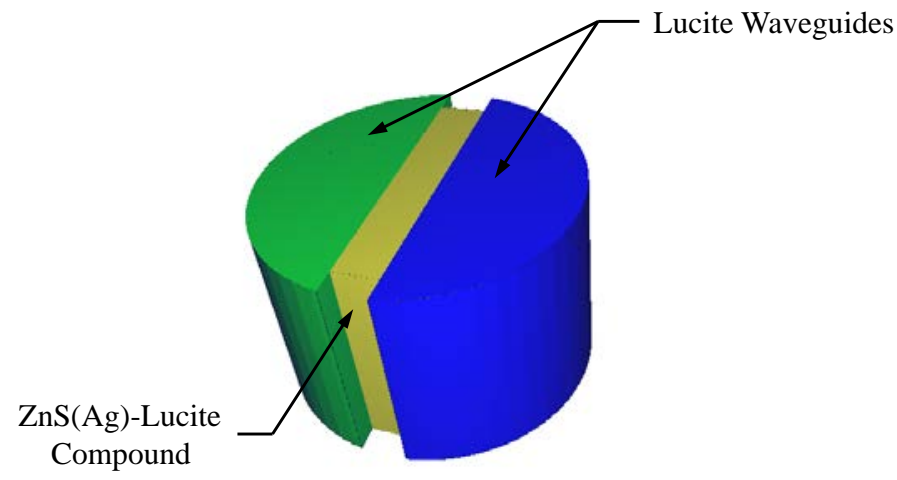

Figure 5. MCNPX-Polimi generated three-dimensional drawing of a TREAT hodoscope fast-neutron scintillation detector.

Once the light producing mechanisms are approximated using MCNPX-PoliMi, these physical values can be used as a starting point for more precise calculations using the Geant4 Simulation Toolkit produced by the European Organization for Nuclear Research (CERN).[8] Geant4 has the ability to fully transport light down to optical energies. Using Geant4 it is possible to more accurately model the physical light generation properties from start to finish. It allows for both a prompt and a de- 
layed component to the scintillation process for a more precise model of a measured digital pulse. It will also allow us take into account the effects of different detector coatings and polishes have on the energy dependent sensitivity of the detectors.

The calculations described in the previous section must rely on heavily-validated models to get the source contributions from a reactor that has been in standby for over 20 years. Isolating the detector response calculations circumvents this dependency, allowing simulations to be benchmarked by easily-accessible laboratory measurements. Such measurements have been performed to benchmark the preliminary detector models using a ${ }^{252} \mathrm{Cf}$ check source and an original hodoscope detector assembly. An example is shown in Figure 6 demonstrating how the light producing mechanisms from recoil electrons from photon interactions are much faster and produce less total light than proton recoils from neutron interactions with hydrogen. These measurements will be used to correlate energy deposition calculations to light output in the detector for post processing of MCNPX-PoliMi simulations and material property definitions in Geant4 models.

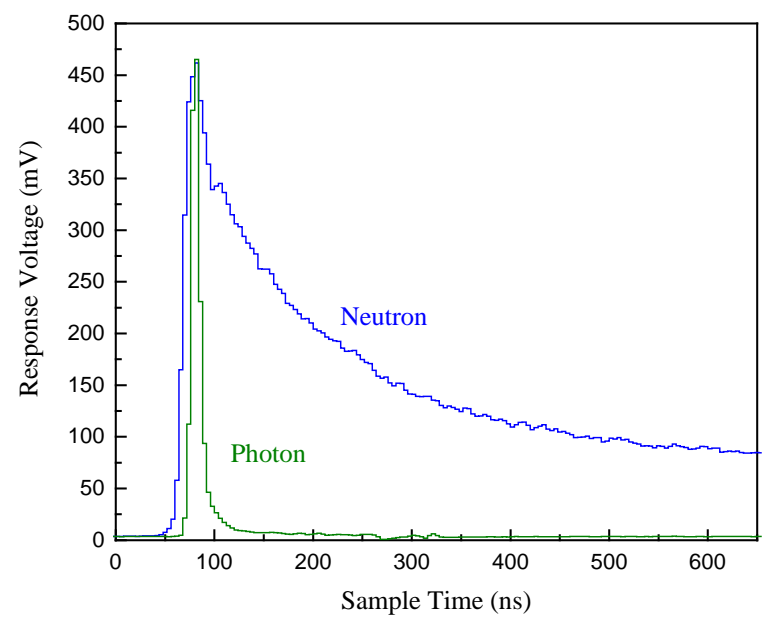

Figure 6. Benchmark digital waveform data recorded using a ${ }^{252} \mathrm{Cf}$ spontaneous fission neutron/gamma source incident upon a TREAT hodoscope fast-neutron detector assembly. Waveforms shown are averages of approximately 1000 pulses.

\section{CONCLUSIONS}

A summary of the preliminary modeling efforts in support of restoration of the TREAT hodoscope for fuel motion monitoring has been provided. The primary goal of this work to date has been to calculate a correlation between time- and location-dependent fissioning mass quantities in the experimental test capsule to a final multichannel detection signal from the hodoscope system. Simulation parameter studies have been initiated to calculate the resulting signal and reactor background fluxes at the point of detection from test fuel under normal operation and during a high-power transient. Simulations have also been initiated to model detector responses to photons and neutrons. These two ongoing modeling efforts will be combined this fiscal year to provide a predictive capability for the hodoscope response and sensitivity in future tests. 


\section{ACKNOWLEDGMENTS}

Funding for this work was provided by the U.S. Department of Energy's Office of Nuclear Energy, from the Nuclear Science User Facilities and the Fuel Cycle Research and Development program.

\section{REFERENCES}

[1] D. C. Crawford, et al., "Experimental Capabilities of the Transient Reactor Test (TREAT) Facility,” Report ANL/RE/CP-96981, Argonne National Laboratory-West, Idaho Falls, Idaho (1998)

[2] M. Corrandini, “Advanced Instrumentation for Transient Reactor Testing,” presentation given at the University of Wisconsin, Madison, Wisconsin. December 17 (2014)

[3] W. F. Hornyak, et al., “A Fast Neutron Detector,” Rev. Sci. Inst. 23, pp. 264-267 (1952)

[4] C. L. Fink, "Optimization of a Hornyak-Button Detector for Fast-Neutron Detection," IEEE Trans. Nucl. Sci. NS-29, pp. 718-721 (1982)

[5] W. C. Kaiser, “System Design Description for TREAT Fast Neutron Hodoscope," Report R0206-100-SA-01, Argonne National Laboratory, Argonne, Illinois (1992)

[6] T. Goorley, et al., “Initial MCNP6 Release Overview”, Nucl. Tech. 180, pp. 298-315 (2012)

[7] E. Padovani, et al., “MCNPX-PoliMi User’s Manual,” C00791 MNYCP, Radiation Safety Information Computational Center, Oak Ridge National Laboratory, Oak Ridge, Tennessee (2012)

[8] J. Allison, et al., “Geant4 Developents and Applications,” IEEE Trans. Nucl. Sci. 53(1), pp. 270-278 (2006) 